\title{
NONLOCAL SYMMETRY AND INTERACTION SOLUTIONS FOR THE NEW (3+1)-DIMENSIONAL INTEGRABLE BOUSSINESQ EQUATION
}

\author{
HengChun Hu* (1) AND XiaOdAn Li
}

\begin{abstract}
The nonlocal symmetry of the new $(3+1)$-dimensional Boussinesq equation is obtained with the truncated Painlevé method. The nonlocal symmetry can be localized to the Lie point symmetry for the prolonged system by introducing auxiliary dependent variables. The finite symmetry transformation related to the nonlocal symmetry of the integrable $(3+1)$-dimensional Boussinesq equation is studied. Meanwhile, the new $(3+1)$-dimensional Boussinesq equation is proved by the consistent tanh expansion method and many interaction solutions among solitons and other types of nonlinear excitations such as cnoidal periodic waves and resonant soliton solution are given.
\end{abstract}

Mathematics Subject Classification. 34A05, 35Q51, 37K40.

Received August 10, 2021. Accepted December 28, 2021.

\section{INTRODUCTION}

The study of nonlinear integrable systems is one of the most important subjects in the nonlinear science. The explicit solutions of nonlinear integrable systems are widely used to explain physical phenomena. Many effective methods are proposed such as the inverse scattering transformation, Bäcklund transformation, Darboux transformation, Painlevé analysis method, the separated variable approach and Hirota bilinear method $[4,6$, $8,14,21,22,26]$, etc. However, it is difficult to find the interaction solutions between a soliton and cnoidal periodic waves for the nonlinear systems with these methods.

Recently, abundant interaction solutions among solitons and different waves including periodic cnoidal waves, Painlevé waves and Boussinesq waves for many integrable systems have been obtained by nonlocal symmetry reduction and the consistent tanh expansion method related to the Painlevé analysis [2, 3, 9, 13, 20]. Lou has pointed out that the residue with respect to the singularity manifold is a nonlocal symmetry for the given truncated Painlevé expansion of the nonlinear Painlevé integrable system [11]. The nonlocal symmetry is a generalized symmetry depending on the integrals of dependent variables [5, 15].

On the other hand, a method called consistent tanh expansion method (CTE) is proposed to identify CTE solvable systems, which is a special simplified form of the consistent Riccati expansion method defined in reference [12]. Based on this method, not only the integrability of nonlinear systems can be clarified but also

Keywords and phrases: New integrable (3+1)-dimensional Boussinesq equation, nonlocal symmetry, consistent tanh expansion method, interaction solution.

College of Science, University of Shanghai for Science and Technology, Shanghai 200093, PR China.

* Corresponding author: hhengchun@163.com 
some interesting interaction solutions between the soliton and many other types of nonlinear waves can be derived $[1,7,10,16-19,23,24]$.

In this paper, we focus on the following new $(3+1)$-dimensional Boussinesq equation

$$
u_{t t}-u_{x x}-\beta\left(u^{2}\right)_{x x}-\gamma u_{x x x x}+\frac{\alpha^{2}}{4} u_{y y}+\alpha u_{y t}+\delta u_{x z}=0,
$$

with $\alpha, \beta, \gamma, \delta$ are nonzero constants. Equation (1.1) is just the extension of the standard Boussinesq equation by adding the term $\frac{\alpha^{2}}{4} u_{y y}+\alpha u_{y t}+\delta u_{x z}$. The new $(3+1)$-dimensional Boussinesq equation describes the propagation of gravity waves over the water surface, in particular the head-on collision of oblique waves. The author has studied the real and complex multiple soliton solutions by means of the simplified Hirota's method and the integrability property of new (3+1)-dimensional Boussinesq equation (1.1) is proved in reference [25].

The paper is organized as follows. In Section 2, the nonlocal symmetry of the new $(3+1)$-dimensional Boussinesq equation is studied by the truncated Painlevé method. In order to solve the initial value problem related to the nonlocal symmetry, the equation (1.1) is extended to the enlarged one by introducing the dependent variables. The finite symmetry transformation is obtained by solving the initial value problem through the Lie's first principle. The multi-solitary wave solution for new (3+1)-dimensional Boussinesq equation is given with the finite symmetry transformation from the nonzero seed solution. In Section 3, some exact interaction solutions for the new (3+1)-dimensional Boussinesq equation (1.1), such as the soliton-cnoidal wave solutions, the resonant solution are studied by the CTE method through the different solutions of the consistent condition. Summary and discussion are given in the last section.

\section{Nonlocal symmetry of the New (3+1)-Dimensional BoussinesQ EQUATION}

In this section, we give the nonlocal symmetry and corresponding finite symmetry transformation for the new (3+1)-dimensional Boussinesq equation (1.1) are discussed based on the truncated Painlevé expansion. Since the nonlocal symmetry includes the arbitrary singularity manifold, we should introduce new dependent variables to make the nonlocal symmetry become the usual Lie point symmetry for the prolonged system.

It is known that the Laurent series form for equation (1.1) reads

$$
u=\frac{u_{0}}{\phi^{2}}+\frac{u_{1}}{\phi}+u_{2}
$$

where the function $\phi=\phi(x, y, z, t)$ is an arbitrary singularity manifold and $u_{0}, u_{1}, u_{2}$ are three functions to be determined later. Substituting the expansion (2.1) into equation (1.1) and vanishing all the coefficients of different powers of $\phi$ independently, we have

$$
\begin{gathered}
u_{0}=-\frac{6 \gamma \phi_{x}^{2}}{\beta}, u_{1}=\frac{6 \gamma \phi_{x x}}{\beta}, \\
u_{2}=\frac{12 \gamma \phi_{x x}^{2}-16 \gamma \phi_{x} \phi_{x x x}-4 \phi_{x}^{2}+\alpha^{2} \phi_{y}^{2}+4 \phi_{t}^{2}+4 \alpha \phi_{y} \phi_{t}+4 \delta \phi_{x} \phi_{z}}{8 \beta \phi_{x}^{2}}, \\
\alpha^{2}\left(K K_{x}+K_{y}\right)+4 \alpha\left(C K_{x}+C_{y}\right)+4\left(C C_{x}+C_{t}\right)+4 \delta Q_{x}-4 \gamma S_{x}=0, \\
u_{2 t t}-u_{2 x x}-\beta\left(u_{2}^{2}\right)_{x x}-\gamma u_{2 x x x x}+\frac{\alpha^{2}}{4} u_{2 y y}+\alpha u_{2 y t}+\delta u_{2 x z}=0,
\end{gathered}
$$


where

$$
S=\frac{\phi_{x x x}}{\phi_{x}}-\frac{3 \phi_{x x}^{2}}{2 \phi_{x}^{2}}, K=\frac{\phi_{y}}{\phi_{x}}, Q=\frac{\phi_{z}}{\phi_{x}}, C=\frac{\phi_{t}}{\phi_{x}}
$$

are invariants of the Möbius transformation. It is clear that equation (2.5) is just equation (1.1) with the solution $u_{2}$ and the residual $u_{1}$ is the symmetry corresponding to the solution $u_{2}$ based on the residual symmetry theorem in [11]. So the truncated Painlevé expansion

$$
u=-\frac{6 \gamma \phi_{x}^{2}}{\beta \phi^{2}}+\frac{6 \gamma \phi_{x x}}{\beta \phi}+u_{2}
$$

is an auto-Bäcklund transformation between the solutions $u$ and $u_{2}$ for the new (3+1)-dimensional Boussinesq equation.

Based on the definition of the residual symmetry, the nonlocal symmetry for equation (1.1) can be read out from the truncated Painlevé expansion

$$
\sigma^{u}=\frac{6 \gamma \phi_{x x}}{\beta}
$$

and the corresponding initial value problem is

$$
\frac{\mathrm{d} \bar{u}}{\mathrm{~d} \epsilon}=\frac{6 \gamma \phi_{x x}}{\beta},\left.\bar{u}\right|_{\epsilon=0}=u,
$$

with $\epsilon$ being an infinitesimal parameter. It is not easy to solve the initial value problem (2.8) according to the Lie first principle due to the intrusion of the arbitrary function $\phi$ its two-order differentiation. To solve the initial value problem related to the nonlocal symmetry (2.7), we localize the nonlocal symmetry to the local Lie point symmetry for the enlarged system by introducing two new dependent variables

$$
\phi_{x}=g, g_{x}=h
$$

It is easily verified that the solution of the symmetry equation for the prolonged system (1.1), (2.3) and (2.9) gives

$$
\sigma^{\phi}=-\phi^{2}, \sigma^{u}=\frac{6 \gamma h}{\beta}, \sigma^{g}=-2 \phi g, \sigma^{h}=-2 g^{2}-2 \phi h .
$$

Then the corresponding initial value problem becomes

$$
\begin{aligned}
& \frac{\mathrm{d} \bar{u}}{\mathrm{~d} \epsilon}=\frac{6 \gamma \bar{h}}{\beta},\left.\bar{u}\right|_{\epsilon=0}=u, \\
& \frac{\mathrm{d} \bar{\phi}}{\mathrm{d} \epsilon}=-\bar{\phi}^{2},\left.\bar{\phi}\right|_{\epsilon=0}=\phi, \\
& \frac{\mathrm{d} \bar{g}}{\mathrm{~d} \epsilon}=-2 \bar{\phi} \bar{g},\left.\bar{g}\right|_{\epsilon=0}=g,
\end{aligned}
$$




$$
\frac{\mathrm{d} \bar{h}}{\mathrm{~d} \epsilon}=-2 \bar{g}^{2}-2 \bar{\phi} \bar{h},\left.\bar{h}\right|_{\epsilon=0}=h .
$$

It can be seen that the nonlocal symmetry (2.7) becomes the Lie point symmetry for the prolonged system (1.1), (2.3) and (2.9) and the corresponding solution of the initial value problem (2.11) can be written as

$$
\begin{gathered}
\bar{\phi}=\frac{\phi}{1+\epsilon \phi}, \bar{g}=\frac{g}{(1+\epsilon \phi)^{2}}, \bar{h}=\frac{h}{(1+\epsilon \phi)^{2}}-\frac{2 g^{2} \epsilon}{(1+\epsilon \phi)^{3}}, \\
\bar{u}=u+\frac{6 \gamma \epsilon h+6 \gamma \epsilon^{2} \phi h-6 \gamma \epsilon^{2} g^{2}}{\beta(1+\epsilon \phi)^{2}}
\end{gathered}
$$

From the finite symmetry transformations (2.12), one can get another solution $\bar{u}$ from a given seed solution $u$. For example, the function

$$
\phi=1+\exp \left(a_{1} x+b_{1} y+c_{1} z+d_{1} t\right)+\exp \left(a_{2} x+b_{2} y+c_{2} z+d_{2} t\right)+\exp \left(a_{3} x+b_{3} y+c_{3} z+d_{3} t\right)
$$

is a solution of the Schwarzian equation (2.4) with the constants selection

$$
a_{2}=a_{3}=a_{1}, d_{1}=d_{3}-\frac{1}{2} \alpha b_{1}+\frac{1}{2} \alpha b_{3}, d_{2}=d_{3}-\frac{1}{2} \alpha b_{2}+\frac{1}{2} \alpha b_{3}
$$

where $a_{1}, b_{1}, b_{2}, b_{3}, c_{1}, c_{2}, c_{3}, d_{3}, \alpha$ are arbitrary constants. Substituting equations (2.13) and (2.14) into equation (2.3), one can obtain the solitary wave solution for equation (1.1) and the tedious expression is omitted here for simplicity. Then taking this solitary wave solution as the seed solution of equation (1.1), we can obtain the two solitoffs interaction solution of the new (3+1)-dimensional Boussinesq equation (1.1) from equations (2.12). The detailed structure is shown in Figure 1 with the constants selection

$$
\alpha=3, \gamma=c_{3}=-1, \beta=\delta=2, \epsilon=0.8, a_{1}=c_{1}=3, b_{1}=b_{2}=b_{3}=c_{2}=d_{3}=2 .
$$

\section{Consistent tanh EXPANSiOn Method AND InteraCtion SOlUtions OF THE NEW $(3+1)$-DIMENSIONAL BOUSSINESQ EQUATION}

It is known that many interaction solutions between solitons and other types of nonlinear waves such as soliton-cnoidal waves, Airy waves, resonant waves for different nonlinear systems can be obtained through the CTE method. In this section, we aim to find new interaction solutions for the new (3+1)-dimensional Boussinesq equation (1.1) with the help of the CTE method by choosing proper function in the consistent condition.

By the leading order analysis for the new (3+1)-dimensional Boussinesq equation (1.1), we can take the following truncated tanh function expansion

$$
u=u_{0}+u_{1} \tanh (\omega)+u_{2} \tanh ^{2}(\omega),
$$

where $u_{0}, u_{1}, u_{2}$ and $\omega$ are functions of $(x, y, z, t)$ to be determined later. Substituting equation (3.1) into equation (1.1) and vanishing the coefficients of different powers of $\tanh (\omega)$, we have

$$
u_{2}=\frac{-6 \gamma \omega_{x}^{2}}{\beta}, u_{1}=\frac{6 \gamma \omega_{x x}}{\beta},
$$




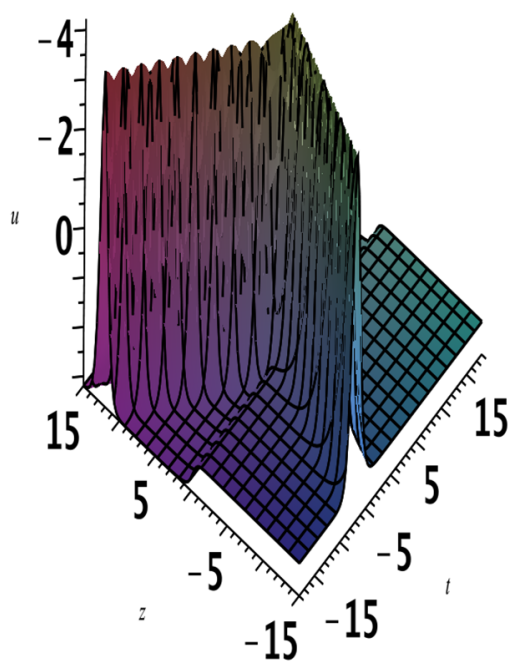

Figure 1. Two solitoffs interaction solution for equation (1.1) with the constants (2.15) at $x=0, y=0$.

$$
u_{0}=\frac{12 \gamma \omega_{x x}^{2}-16 \gamma \omega_{x} \omega_{x x x}+4 \omega_{t}^{2}-4 \omega_{x}^{2}+4 \alpha \omega_{y} \omega_{t}+32 \gamma \omega_{x}^{4}+\alpha^{2} \omega_{y}^{2}+4 \delta \omega_{x} \omega_{z}}{8 \beta \omega_{x}^{2}}
$$

and the function $\omega$ should satisfy the following condition

$$
\begin{aligned}
16 \gamma \omega_{x} \omega_{x x} \omega_{x x x} & -4 \alpha \omega_{x x} \omega_{y} \omega_{t}-4 \omega_{x x} \omega_{t}^{2}-4 \delta \omega_{x} \omega_{x x} \omega_{z}-4 \gamma \omega_{x}^{2} \omega_{x x x x}-12 \gamma \omega_{x x}^{3}-\alpha^{2} \omega_{x x} \omega_{y}^{2} \\
& +\alpha^{2} \omega_{x}^{2} \omega_{y y}+4 \alpha \omega_{x}^{2} \omega_{y t}+4 \omega_{x}^{2} \omega_{t t}+16 \gamma \omega_{x}^{4} \omega_{x x}+4 \delta \omega_{x}^{2} \omega_{x z}=0,
\end{aligned}
$$

which is called the consistent condition for the new (3+1)-dimensional Boussinesq equation (1.1). It is very difficult to solve the nonlinear equation (3.3) because of the higher derivatives of the unknown function $\omega$. From equations (3.1)-(3.3), we can prove the following nonauto-Bäcklund transformation (BT) theorem after direct calculation.

Nonauto-BT theorem: If $\omega$ is a solution of the consistent condition (3.3), then

$$
\begin{aligned}
u= & \frac{12 \gamma \omega_{x x}^{2}-16 \gamma \omega_{x} \omega_{x x x}+4 \omega_{t}^{2}-4 \omega_{x}^{2}+4 \alpha \omega_{y} \omega_{t}+32 \gamma \omega_{x}^{4}+\alpha^{2} \omega_{y}^{2}+4 \delta \omega_{x} \omega_{z}}{8 \beta \omega_{x}^{2}} \\
& +\frac{6 \gamma \omega_{x x}}{\beta} \tanh (\omega)-\frac{6 \gamma \omega_{x}^{2}}{\beta} \tanh ^{2}(\omega),
\end{aligned}
$$

is a consistent tanh expansion solution of equation (1.1). That is to say, once the solution of equation (3.3) is known, the corresponding expression $u$ with equation (3.4) for equation (1.1) can be obtained from the nonauto-BT theorem directly. Some interesting examples are listed in the following paper.

\subsection{Simple soliton solution}

A quite trivial line solution of the consistent condition (3.3) has the form

$$
\omega=a x+b y+c z+d t+e,
$$


where $a, b, c, d, e$ are arbitrary constants. Substituting the trivial line solution (3.5) into equation (3.4), the simple traveling wave solution of the new (3+1)-dimensional Boussinesq equation (1.1) yields

$$
u=\frac{4 d^{2}-4 a^{2}+4 \alpha b d+4 \delta a c+32 \gamma a^{4}+\alpha^{2} b^{2}}{8 \beta a^{2}}-\frac{6 \gamma a^{2}}{\beta} \tanh ^{2}(a x+b y+c z+d t+e) .
$$

\subsection{Interaction solutions between the soliton and cnoidal periodic waves}

In order to derive the soliton-cnoidal wave solution for equation (1.1), the solution of (3.3) should be assumed as the following form:

$$
\begin{aligned}
\omega & =k_{1} x+l_{1} y+p_{1} z+q_{1} t+G\left(k_{2} x+l_{2} y+p_{2} z+q_{2} t\right) \\
& \equiv k_{1} x+l_{1} y+p_{1} z+q_{1} t+G(X) .
\end{aligned}
$$

After substituting (3.6) into the consistent condition (3.3), the function $G(X)$ should be a solution of the following equation

$$
\begin{aligned}
& G_{1 X}^{2}(X)=a_{0}+a_{1} G_{1}(X)+a_{2} G_{1}^{2}(X)+a_{3} G_{1}^{3}(X)+a_{4} G_{1}^{4}(X), \\
& G_{1}(X)=G_{X}(X),
\end{aligned}
$$

where the coefficients $a_{i}(i=1,2,3,4)$ are

$$
\left\{\begin{aligned}
a_{0}= & \frac{1}{12 \gamma k_{2}^{6}}\left(12 \gamma a_{2} k_{1}^{2} k_{2}^{4}-24 \gamma a_{3} k_{1}^{3} k_{2}^{3}+144 \gamma k_{1}^{4} k_{2}^{2}-\alpha^{2} l_{1}^{2} k_{2}^{2}-4 \alpha^{2} l_{1} l_{2} k_{1} k_{2}+5 \alpha^{2} l_{2}^{2} k_{1}^{2}\right. \\
& -4 \alpha l_{1} q_{1} k_{2}^{2}-8 \alpha l_{1} q_{2} k_{1} k_{2}-8 \alpha l_{2} q_{1} k_{1} k_{2}+20 \alpha l_{2} q_{2} k_{1}^{2}-12 \delta p_{1} k_{1} k_{2}^{2}-4 q_{1}^{2} k_{2}^{2} \\
& \left.+12 \delta p_{2} k_{1}^{2} k_{2}-16 q_{1} q_{2} k_{1} k_{2}+20 q_{2}^{2} k_{1}^{2}\right) \\
a_{1}= & \frac{1}{2 \gamma k_{2}^{5}}\left(4 \gamma a_{2} k_{1} k_{2}^{4}-6 \gamma a_{3} k_{1}^{2} k_{2}^{3}+32 \gamma k_{1}^{3} k_{2}^{2}-\alpha^{2} l_{1} l_{2} k_{2}+\alpha^{2} l_{2}^{2} k_{1}-2 \alpha l_{1} q_{2} k_{2}-2 \alpha l_{2} q_{1} k_{2}\right. \\
& \left.+4 \alpha l_{2} q_{2} k_{1}-2 \delta p_{1} k_{2}^{2}+2 \delta p_{2} k_{1} k_{2}-4 q_{1} q_{2} k_{2}+4 q_{2}^{2} k_{1}\right), \\
a_{4}= & 4
\end{aligned}\right.
$$

which results in the following solutions:

$$
\begin{aligned}
u= & -\frac{6 \gamma\left(k_{1}+k_{2} G_{1}\right)^{2}}{\beta} \tanh ^{2}(\omega)+\frac{6 \gamma k_{2}^{2} G_{1 X}}{\beta} \tanh (\omega)+\frac{1}{8 \beta\left(k_{1}+k_{2} G_{1}\right)^{2}}\left[4\left(q_{1}+q_{2} G_{1}\right)^{2}\right. \\
& +12 \gamma k_{1}^{4} G_{1 X}^{2}-4\left(k_{1}+k_{2} G_{1}\right)^{2}+4 \alpha\left(q_{1}+q_{2} G_{1}\right)\left(l_{1}+l_{2} G_{1}\right)-16 \gamma k_{2}^{3}\left(k_{1}+k_{2} G_{1}\right) G_{1 X X} \\
& \left.+32\left(k_{1}+k_{2} G_{1}\right)^{4}+\alpha^{2}\left(l_{1}+l_{2} G_{1}\right)^{2}+4 \delta\left(k_{1}+k_{2} G_{1}\right)\left(p_{1}+p_{2} G_{1}\right)\right] .
\end{aligned}
$$

The solution (3.9) can be regarded as the interaction solution between the soliton solution and the $G$ function solution. It is obvious that the general solution for equation (3.7) is defined by elliptic function, that is, we can select the proper elliptic function to construct the expression of $\omega$, thus the solution for the new (3+1)dimensional Boussinesq equation (1.1) can be obtained. For example, one simple solution of equation (3.7) is

$$
G_{1}=\mu_{0}+\mu_{1} \operatorname{sn}(m X, n)
$$


where $\operatorname{sn}(m X, n)$ is the usual Jacobi elliptic sine function. Then substituting equation (3.8) and equation (3.10) into equation (3.7), we can find

$$
\left\{\begin{aligned}
\mu_{1}= & \frac{\left(k_{1}+k_{2} \mu_{0}\right) n}{k_{2}}, \\
m= & \frac{2\left(k_{1}+k_{2} \mu_{0}\right)}{k_{2}}, \\
q_{1}= & \frac{2 q_{2} k_{1}+\alpha l_{2} k_{1}-\alpha l_{1} k_{2}}{2 k_{2}}, \\
p_{1}= & \frac{1}{\delta k_{2}}\left(8 \gamma k_{2}^{4} \mu_{0}^{3}+24 \gamma k_{1} k_{2}^{3} \mu_{0}^{2}+24 \gamma \mu_{0} k_{1}^{2} k_{2}^{2}+\delta p_{2} k_{1}+8 \gamma k_{1}^{3} k_{2}-8 \gamma n^{2} k_{1}^{3} k_{2}\right. \\
& \left.-24 \gamma \mu_{0} n^{2} k_{1}^{2} k_{2}^{2}-24 \gamma \mu_{0}^{2} n^{2} k_{1} k_{2}^{3}-8 \gamma \mu_{0}^{3} n^{2} k_{2}^{4}\right), \\
a_{2}= & \frac{4\left(5 k_{2}^{2} \mu_{0}^{2}-k_{1}^{2}-k_{1}^{2} n^{2}-2 k_{1} k_{2} \mu_{0} n^{2}-2 k_{1} k_{2} \mu_{0}-k_{2}^{2} \mu_{0}^{2} n^{2}\right)}{k_{2}^{2}} \\
a_{3}= & -16 \mu_{0} .
\end{aligned}\right.
$$

The integral of equation (3.10) is

$$
G=\mu_{0} X+\mu_{1} \int_{X_{0}}^{X} \operatorname{sn}(m Y, n) \mathrm{d} Y .
$$

Then substituting equations (3.10)-(3.12) into the solution (3.9), the soliton-cnoidal wave interaction solution for equation (1.1) can be obtained.

In order to find more interaction solutions between soliton and other nonlinear excitations of equation (1.1), one can select the more proper function $\omega$ which satisfies the consistent condition (3.3). The first type of the soliton-cnoidal wave interaction solution for equation (1.1) can be obtained by fixing the function $\omega$ as

$$
\omega=a_{0} x+b_{0} y+c_{0} z+d_{0} t+\frac{1}{2} \operatorname{arctanh}\left[m \operatorname{sn}\left(a_{1} x+b_{1} y+c_{1} z+d_{1} t, m\right)\right]
$$

Then substituting equation (3.13) into the consistent condition (3.3), we can find the constant relation

$$
a_{0}=\frac{1}{2} a_{1}, c_{0}=-\frac{2 \gamma m^{2} a_{1}^{3}-2 \gamma a_{1}^{3}-\delta c_{1}}{2 \delta}, d_{0}=-\frac{1}{2} \alpha b_{0}+\frac{1}{4} \alpha b_{1}+\frac{1}{2} d_{1},
$$

and the constants $b_{0}, a_{1}, b_{1}, c_{1}, d_{1}, m$ are arbitrary constants. So the first type of the soliton-cnoidal wave interaction solution for equation (1.1) can be obtained by substituting equation (3.13) into equation (3.4) with the constant constraint (3.14).

The second type of the soliton-cnoidal wave interaction solution for equation (1.1) can be obtained by fixing the function $\omega$ as

$$
\omega=\frac{a_{0} x+b_{0} y+c_{0} z+d_{0} t}{e_{0}}+A \operatorname{arctanh}\left[\operatorname{sn}\left(\frac{a_{1} x+b_{1} y+c_{1} z+d_{1} t}{e_{1}}, m\right)\right] .
$$

Then substituting equation (3.15) into the consistent condition (3.3), we can find the constant relation

$$
a_{1}=0, d_{1}=-\frac{1}{2} \alpha b_{1}
$$




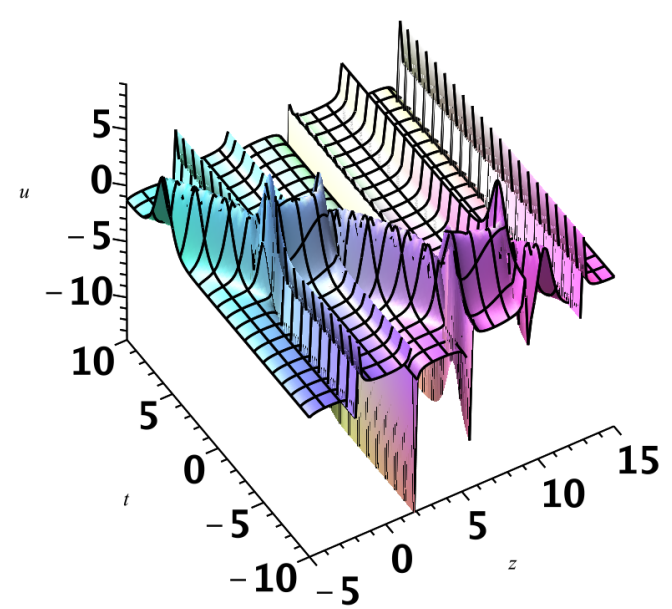

Figure 2. The second type of the soliton-cnoidal wave interaction solution (3.4) for equation (1.1) with equations (3.15)-(3.16) the constants $\alpha=\beta=\gamma=\delta=2, A=0.5, m=0.8, a_{0}=$ $2.2, b_{0}=1, b_{1}=c_{0}=c_{1}=d_{0}=e_{0}=2, e_{1}=5, y=0, z=0$.

and the constants $A, a_{0}, b_{0}, b_{1}, c_{0}, c_{1}, d_{0}, m$ are arbitrary constants. So the first type of the soliton-cnoidal wave interaction solution for equation (1.1) can be obtained by substituting equation (3.15) into equation (3.4) with the constant constraint (3.16). The structure of the second type of the soliton-cnoidal wave interaction solution is given in Figure 2.

The third type of soliton-cnoidal interaction solution for equation (1.1) can be obtained by choosing the function $\omega$ as

$$
\omega=a_{0} x+b_{0} y+c_{0} z+d_{0} t+A E_{f}\left(\operatorname{sn}\left(a_{1} x+b_{1} y+c_{1} z+d_{1} t, m\right), n\right),
$$

where $E_{f}$ is the first type of incomplete elliptic integral. Substituting equation (3.17) into equation (3.3), the constants should satisfy the relation $m=n$ and other constants are arbitrary. Then substituting equation (3.17) and $m=n$ into equation (1.1), one can obtain the third type of soliton-cnoidal interaction solution for equation (1.1) with the proper parameter selection.

\subsection{Resonant soliton solution}

One special resonant soliton solution for the new $(3+1)$-dimensional Boussinesq equation (1.1) can be obtained by choosing

$$
\omega=a_{0} x+b_{0} y+c_{0} z+d_{0} t+C \ln \left(1+\exp \left(a_{1} x+b_{1} y+c_{1} z+d_{1} t\right)\right)
$$

by substituting equation (3.18) into the consistent condition (3.3), the constants should satisfy the relation

$$
\begin{aligned}
C & =\frac{1}{2}, b_{0}=\frac{\alpha a_{0} b_{1}+2 a_{0} d_{1}-2 a_{1} d_{0}+2 \sqrt{-3 \gamma a_{0}^{2} a_{1}^{2}\left(2 a_{0}+a_{1}\right)^{2}}}{\alpha a_{1}}, \\
c_{0} & =\frac{a_{0} a_{1}\left(16 \gamma a_{0}^{2} a_{1}+12 \gamma a_{0} a_{1}^{2}+2 \gamma a_{1}^{3}+\delta c_{1}\right)-\left(2 d_{1}+\alpha b_{1}\right) \sqrt{-3 \gamma a_{0}^{2} a_{1}^{2}\left(2 a_{0}+a_{1}\right)^{2}}}{\delta a_{1}^{2}},
\end{aligned}
$$




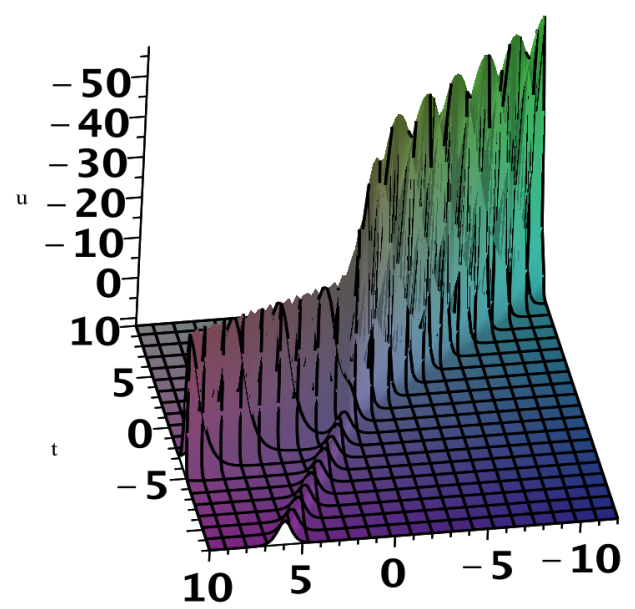

FiguRE 3. The resonant soliton solution (3.4) for equation (1.1) with equations (3.18)-(3.20) at $y=0, z=0$.

where $a_{0}, d_{0}, a_{1}, b_{1}, c_{1}, d_{1}$ are arbitrary constants. The special resonant solution has been given out graphically in Figure 3 with the constants selection

$$
\alpha=3, \beta=1, \gamma=-1, \delta=2, a_{0}=1, a_{1}=4.8, b_{1}=2, c_{1}=2, d_{0}=d_{1}=2 .
$$

\section{Summary AND DISCUSSION}

In summary, the nonlocal symmetry of the new $(3+1)$-dimensional Boussinesq equation is obtained with the truncated Painlevé expansion method. In order to solve the initial value problem related to the nonlocal symmetry, we prolong the new $(3+1)$-dimensional Boussinesq equation such that nonlocal symmetry can become the local Lie point symmetry for the prolonged system. The finite symmetry transformation of the prolonged system is derived by using Lie's first principle and two solitoffs interaction solution for the equation (1.1) is obtained by choosing the proper solution of the consistent condition. Meanwhile, the new (3+1)-dimensional Boussinesq equation is proved to be CTE solvable and we find abundant interaction solutions between the soliton and cnoidal-periodic waves with arbitrary constants. We hope that many other integrable properties and new soliton solutions for the new (3+1)-dimensional Boussinesq equation (1.1) are worthy of study in future.

Acknowledgements. The work is supported by National Natural Science Foundation of China (No.11471215), Shanghai Natural Science Foundation (No.18ZR142600).

\section{REFERENCES}

[1] C.L. Chen and S.Y. Lou, CTE solvability and exact solution to the Broer-Kaup system. Chin. Phys. Lett. 30 (2013) 110202.

[2] X.P. Cheng, S.Y. Lou, C.L. Chen and X.Y. Tang, Interactions between solitons and other nonlinear Schrödinger waves. Phys. Rev. E 89 (2014) 1-14.

[3] W.G. Cheng, B. Li and Y. Chen, Nonlocal symmetry and exact solutions of the (2+1)-dimensional breaking soliton equation. Commun. Nonlinear Sci. Numer. Simulat. 29 (2015) 198-207.

[4] C.S. Gardner, J.M. Greene, M.D. Kruskal and R.M. Miura, Method for solving the Korteweg-de Vries equation. Phys. Rev. Lett. 19 (1967) 1095-1097.

[5] G.A. Guthrie, More non-local symmetries of the KdV equation. J. Phys. A: Math. Gen. 26 (1993) L905-L908.

[6] R. Hirota, The Direct Method in Soliton Theory. Cambridge University Press, Cambridge (2004).

[7] X.R. Hu and Y. Chen, Nonlocal symmetries, consistent Riccati expansion integrability, and their applications of the (2+1)dimensional Broer-Kaup-Kupershmidt system. Chin. Phys. B 24 (2015) 090203. 
[8] H.C. Hu, X. Hu and B.F. Feng, Nonlocal symmetry and consistent tanh expansion method for the coupled integrable dispersionless equation. Z. Naturforsch. A 71 (2016) 235-240.

[9] X.R. Hu, S.Y. Lou and Y. Chen, Explicit solutions from eigenfunction symmetry of the Korteweg-de Vries equation. Phys. Rev. E 85 (2012) 056607.

[10] Y.Y. Li and H.C. Hu, Nonlocal symmetries and interaction solutions of the Benjamin-Ono equation. Appl. Math. Lett. 75 (2018) 18-23.

[11] S.Y. Lou, Residual symmetries and Bäcklund transformations. Preprint arXiv:1308.1140v1 [nlin.SI] (2013).

[12] S.Y. Lou, Consistent Riccati expansion for integrable systems. Stud. Appl. Math. 134 (2015) 372-402.

[13] S.Y. Lou, X.R. Hu and Y. Chen, Nonlocal symmetries related to Bäcklund transformation and their applications. J. Phys. A: Math. Theor. 45 (2012) 155209.

[14] V.B. Matveev, and M.A. Salle, Darboux Transformations and Solitons, Springer-Verlin, Berlin (1991).

[15] P.J. Olver, J. Sanders and J.P. Wang, Ghost symmetries. J. Nonlinear Math. Phys. 20 (2002) 164-172.

[16] B. Ren, Interaction solutions for mKP equation with nonlocal symmetry reductions and CTE method. Phys. Scr. 90 (2015) 065206.

[17] B. Ren, Symmetry reduction related with nonlocal symmetry for Gardner equation. Commun. Nonlinear Sci. Numer. Simulat. 42 (2017) 456-463.

[18] B. Ren, W.X. Ma and J. Yu, Characteristics and interactions of solitary and lump waves of a $(2+1)$-dimensional coupled nonlinear partial differential equation. Nonlinear Dyn. 96 (2019) 717-727.

[19] B. Ren, J. Lin and Z.M. Lou, Consistent Riccati expansion and rational solutions of the Drinfel'd-Sokolov-Wilson equation. Appl. Math. Lett. 105 (2020) 106326.

[20] B. Ren, X.P. Cheng and J. Lin, The (2+1)-dimensional Konopelchenko-Dubrovsky equation: nonlocal symmetries and interaction solutions. Nonlinear Dyn. 86 (2016) 1855-1862.

[21] C. Rogers and W.K. Schief, Bäcklund and Darboux Transformation, Geometry and Modern Applications in Soliton Theory, Cambridge University Press, Cambridge (2002).

[22] X.Y. Tang, S.Y. Lou and Y. Zhang, Localized excitations in (2+1)-dimensional systems. Phys. Rev. E 66 (2002) 046601.

[23] Y.H. Wang, CTE method to the interaction solutions of Boussinesq-Burgers equations. Appl. Math. Lett. 38 (2014) $100-105$.

[24] Y.H. Wang and H. Wang, Symmetry analysis and CTE solvability for the (2+1)-dimensional Boiti-Leon-Pempinelli equation. Phys. Scr. 89 (2014) 125203.

[25] A.M. Wazwaz and L. Kaur, New integrable Boussinesq equations of distinct dimensions with diverse variety of soliton solutions. Nonlinear Dyn. 97 (2019) 83-94.

[26] J. Weiss, M. Tabor and G. Carnevale, The Painlevé property for partial differential equations. J. Math. Phys. 24 (1983) $522-526$.

\section{Subscribe to Open (S2O) A fair and sustainable open access model}

This journal is currently published in open access under a Subscribe-to-Open model (S2O). S2O is a transformative model that aims to move subscription journals to open access. Open access is the free, immediate, online availability of research articles combined with the rights to use these articles fully in the digital environment. We are thankful to our subscribers and sponsors for making it possible to publish this journal in open access, free of charge for authors.

\section{Please help to maintain this journal in open access!}

Check that your library subscribes to the journal, or make a personal donation to the S2O programme, by contacting subscribers@edpsciences.org

More information, including a list of sponsors and a financial transparency report, available at: https://www.edpsciences.org/en/maths-s2o-programme 